\title{
Silicon Oxide Surface Segregation in CO Oxidation on Pd: An in situ PEEM, MS and XPS Study
}

\author{
D. Vogel $\cdot$ Z. Budinska $\cdot$ C. Spiel $\cdot$ R. Schlögl $・$ \\ Y. Suchorski · G. Rupprechter
}

Received: 30 October 2012/ Accepted: 12 December 2012/Published online: 8 January 2013

(C) The Author(s) 2013. This article is published with open access at Springerlink.com

\begin{abstract}
The effect of silicon oxide surface segregation on the locally-resolved kinetics of the $\mathrm{CO}$ oxidation reaction on individual grains of a polycrystalline Pd foil was studied in situ by PEEM, MS and XPS. The silicon oxide formation induced by Si-impurity segregation at oxidizing conditions, was monitored by XPS and its impact on the global and local (spatially resolved) kinetics of the $\mathrm{CO}$ oxidation was determined by MS and PEEM. The results reveal a drastic inhibiting effect of silicon oxide on the Pd reactivity towards $\mathrm{CO}$ oxidation, manifested both in the collapse of the global $\mathrm{CO}_{2}$ formation rate and in the modified local reactive properties of individual Pd micrograins. The presence of adsorbed oxygen on the Pd surface effectively enhances the silicon segregation to the $\mathrm{Pd}$ surface.
\end{abstract}

Keywords CO oxidation - Polycrystalline Pd foil $\cdot \mathrm{Si}$ segregation $\cdot \mathrm{Si}$ oxide formation $\cdot$ Photoemission electron microscopy $\cdot \mathrm{X}$-ray photoelectron spectroscopy

\section{Introduction}

Catalytic CO oxidation on Pt-group metal surfaces is often considered as a simple model reaction of significant practical meaning with respect to pollution emission control.

D. Vogel · Z. Budinska · C. Spiel · Y. Suchorski ( $₫)$.

G. Rupprechter

Institute of Materials Chemistry, Vienna University

of Technology, Getreidemarkt 9, 1060 Vienna, Austria

e-mail: yuri.suchorski@imc.tuwien.ac.at

D. Vogel · R. Schlögl

Fritz-Haber-Institut der Max-Planck-Gesellschaft,

Faradayweg 4-6, 14195 Berlin, Germany
More than three decades ago, pioneering molecular beam experiments of $\mathrm{CO}$ oxidation on $\mathrm{Pd}(111)$ surfaces performed by Engel and Ertl provided first evidence that the reaction follows the Langmuir-Hinshelwood mechanism [1]. Further applications of the surface science approach to single crystal surfaces revealed the elementary steps of this reaction such as molecular adsorption of $\mathrm{CO}$, dissociative adsorption of oxygen, surface reaction and desorption of carbon dioxide, and culminated in the Nobel Prize awarded to Gerhard Ertl in 2007 [2].

However, at the same time this seemingly simple model reaction was found to be quite complex in case of real catalysts consisting of oxide supported precious metal nanoparticles, and bridging the "materials and pressure gap" between the single crystal surfaces in UHV and real catalysts under ambient conditions is still a challenging task. To overcome the "material gap" between single crystal surfaces and real catalysts, many approaches have been applied, e.g. the investigation of field emitter tips [3], cylindrical single crystals [4] or polycrystalline foils [5-7].

A polycrystalline foil is a particularly suitable model system since it exhibits $\mu \mathrm{m}$-sized domains of different orientations which are automatically exposed to exactly the same external parameters such as partial pressures and temperature. This means that precise comparative studies of the catalytic behavior of individual (hkl) orientations, such as the role of surface oxide formation can be performed, provided the applied experimental technique is able to read out the information from the $\mu \mathrm{m}$-sized domains. Our new experimental approach based on photoemission electron microscopy (PEEM) exploits the parallel imaging principle of PEEM and uses the digital analysis of in situ recorded video-PEEM files allowing thus laterally resolved kinetic measurements on a $\mu \mathrm{m}$-scale $[7,8]$. We have already applied this approach to study the 
local kinetics of the $\mathrm{CO}$ oxidation on individual grains of polycrystalline Pt [7, 9] and Pd [10] foil and to directly compare the behavior of reaction-diffusion fronts on differently oriented domains [8,9].

New experimental developments of surface sensitive techniques applicable under high pressure conditions (i.e. mbar to atmospheric pressure), such as high-pressure scanning tunnelling microscopy (HP-STM), polarization modulation infrared reflection absorption spectroscopy (PM-IRAS) and sum-frequency generation (SFG) have provided insights to catalytic reactions such as $\mathrm{CO}$ oxidation under more realistic catalytic conditions (bridging the "pressure gap" [11-16]). In some studies, (surface) oxide formation was observed in parallel with an activity increase and a Mars-van-Krevelen like mechanism has been proposed for the CO oxidation reaction on $\mathrm{Pt}$ and Pd surfaces at elevated pressures [16, 17]. Despite an intensive debate about the reaction mechanism and about the nature of the most active phase in $\mathrm{CO}$ oxidation $[18,19]$, the LangmuirHinshelwood mechanism seems still to predict well the kinetics of the $\mathrm{CO}$ oxidation reaction on Pt-group metals at both low and high pressures [12]: the O-covered metal surface responsible for the high catalytic activity in UHV, appears also as the most active phase at elevated pressures, unless oxide formation initiates the deactivation, especially for $\mathrm{Pd}$ and $\mathrm{Rh}$ surfaces.

Despite of the intensive studies of the role of palladium oxides in CO oxidation on $\mathrm{Pd}$ [20], much less attention is directed to the role of "non-palladium" oxides on the Pd surface in the CO oxidation. Recently it was shown, that such "stranger" oxides present on the platinum-metal surface can significantly influence the reaction, even at small oxide coverages $[21,22]$. It is also known that impurities commonly present in commercially used catalysts, such as $\mathrm{Si}$, might form surface oxides under oxidizing atmosphere [23], but, to our knowledge, aimed studies of such oxides under reaction conditions are very scarce. In the present contribution we apply PEEM combined with in situ $\mathrm{x}$-ray photoelectron spectroscopy (XPS) and mass-spectrometry (MS) to study the role of the surface oxides formed by Si impurities in the kinetics of the $\mathrm{CO}$ oxidation on $\mathrm{Pd}$.

\section{Experimental}

The experiments were performed in a UHV system consisting of two independently operated chambers connected with each other by a sample transfer line, thus allowing a common reactive gas atmosphere in the $10^{-4}-10^{-9}$ mbar range. The "microscopy" chamber is equipped with a PEEM (Staib Instruments), an MS (MKS Instruments), a LEED system (Omicron), a high purity gas supply system $\left(\mathrm{O}_{2}\right.$ : $\left.99.999 \%, \mathrm{CO}: 99.97 \%\right)$ and sample preparation facilities for cleaning the sample by argon ion sputtering and subsequent annealing. The "spectroscopy" chamber is equipped with an XPS-system (Phoibos 100 hemispherical energy analyzer and XR 50 twin anode X-ray source, SPECS). The general configuration of the experimental setup and a scheme of the experiment are shown in Fig. 1.

The investigated sample, a $10 \times 10 \mathrm{~mm}^{2}$ polycrystalline Pd foil (AlfaAesar, $99.9 \%$ purity), consists mainly of [100]-, [110]- and [111]-oriented grains which were formed after annealing the foil for several hours at $1100 \mathrm{~K}$ in UHV. The sample temperature was measured by a $\mathrm{NiCr} /$ $\mathrm{Ni}$ thermocouple spot-welded to the front side of the $\mathrm{Pd}$ sample. Prior to catalytic measurements, the surface was repeatedly $\mathrm{Ar}^{+}$sputtered and annealed at $1073 \mathrm{~K}$.

The principle of the local kinetic measurements by PEEM has been described in detail before [7-9] and is therefore summarized here only briefly (see also Fig. 1). The CO oxidation reaction on the Pd foil is monitored simultaneously by MS and by PEEM, whereby MS provides the global $\mathrm{CO}_{2}$ rate produced by the whole polycrystalline sample. PEEM, in turn, provides the laterally resolved kinetic information from the individual grains.

The PEEM image which is formed by photoelectrons created by UV light illumination of the sample (with a deuterium discharge lamp, $\mathrm{E}_{\max } \sim 6.5 \mathrm{eV}$ ) reflects the lateral distribution of the local work function across the sample. This allows for differentiation between grains of different surface orientation and between different adsorbates by correlation of the local image intensity with the work function values of the corresponding clean and adsorbate-covered single crystals [7]. The PEEM-image is recorded in situ with a high-speed CCD-camera (Hamamatsu), so the intensity of a selected region-of-interest,

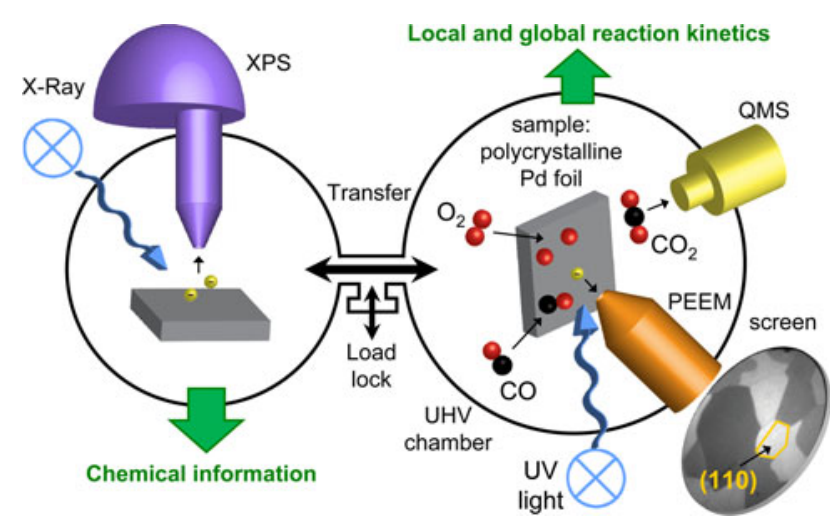

Fig. 1 Configuration of the experimental setup and scheme of the experiment. The simultaneous monitoring of the ongoing $\mathrm{CO}$ oxidation by PEEM and MS provides information on kinetic transitions on the whole sample (global MS measurements) and on the individual grains of a polycrystalline Pd foil (local PEEM measurements). The XPS analysis provides data on the chemical composition of the samples in UHV and under the same gas atmosphere as in PEEM 
i.e. one particular grain, can be monitored during a typical CO pressure scan. The XPS analysis provides data on the chemical composition of the samples in UHV or under the same gas atmosphere as in PEEM.

\section{Results and Discussion}

\subsection{Global and Local Reaction Kinetics}

To correlate the kinetic transitions in CO oxidation reaction on individual grains on the polycrystalline $\mathrm{Pd}$ foil with the averaged (global) kinetics of the whole sample, the orientation of the individual grains was established first, and global kinetic studies in the bistability range of $\mathrm{CO}$ oxidation were performed. The results are summarized in Fig. 2: the upper row (Fig. 2a-c) shows PEEM-frames of the clean $\left(\mathrm{Ar}^{+}\right.$ sputtered and annealed) Pd foil (Fig. 2a), the same surface covered by adsorbed oxygen (Fig. 2b) and again the same area but after oxygen treatment at $973 \mathrm{~K}$ and $5 \times 10^{-6} \mathrm{mbar}$ (Fig. 2c). In Fig. 2a three individual [110]-, [100]- and [111]oriented grains are indicated, whose crystallographic orientation has been determined by the differences in contrast, as described in detail in Ref. [7]. The lower plots in Fig. 2 show the global (MS-measured for the whole sample) $\mathrm{CO}_{2}$ reaction rate recorded during an isothermal cyclic $\mathrm{CO}$ pressure scan at constant oxygen pressure of $1.3 \times 10^{-5} \mathrm{mbar}$ for the clean, i.e. sputtered and annealed Pd surface (Fig. 2d), and for the same surface which was additionally treated by oxygen at $\mathrm{po}_{2}=5 \times 10^{-6}$ mbar for $15 \mathrm{~min}$ at three different temperatures of 873,973 and $1073 \mathrm{~K}$, also in comparison with the clean Pd surface (Fig. 2e).

Typically for $\mathrm{CO}$ oxidation on $\mathrm{Pd}$, the surface in the high reactivity steady state (predominantly oxygen-covered), exhibits an almost linear increase of the $\mathrm{CO}_{2}$ production rate with increasing $\mathrm{CO}$ pressure, till a kinetic transition from the high reactivity state to a low reactivity state occurs (Fig. 2d). This is the result of the collapsing oxygen adsorption due to the CO-induced poisoning (inhibition of dissociative oxygen adsorption, [2]) of the $\mathrm{Pd}$ surface. The corresponding transition point is termed $\tau_{\mathrm{A}}$ whereas the point of the reverse transition is called $\tau_{\mathrm{B}}$ (Fig. 2d). Because of the effective blocking of adsorption sites for oxygen by $\mathrm{CO}$ the reverse transition $\tau_{\mathrm{B}}$ from the inactive to the active state occurs at a lower $\mathrm{CO}$ partial pressure than the transition $\tau_{\mathrm{A}}$, resulting in the hysteresis in the $\mathrm{CO}_{2}$ production rate as seen in Fig. $2 \mathrm{~d}$ for the sputtered and annealed Pd surface and in Fig. 2e for the Pd surface treated at $873 \mathrm{~K}$ in oxygen at $5 \times 10^{-6}$ mbar. Such hysteresis-like behavior of the $\mathrm{CO}$ oxidation reaction is called bistability since two states of the reaction system are possible at the same parameter set depending on the system's prehistory (for details see refs. [21, 22]).
For the Pd surface treated by oxygen at $873 \mathrm{~K}$ the $\mathrm{CO}_{2}$ rate appears considerably lower, but the hysteresis is still present (Fig. 2e). Oxygen treatment at higher temperatures causes further decrease of the $\mathrm{CO}_{2}$ rate (e.g. for $973 \mathrm{~K}$, Fig. 2e) and even a disappearance of the hysteresis (see the $1073 \mathrm{~K}$ curve in Fig. 2e).

For the laterally resolved kinetics of the $\mathrm{CO}$ oxidation, the video-PEEM sequences (recorded simultaneously with the MS monitoring) were analyzed. Figure 3 a shows an example of such analysis where the local PEEM intensity for a particular domain, in the present case a $\operatorname{Pd}(110)$ domain, is shown in analogy to the globally MS-measured $\mathrm{CO}_{2}$ rate in Fig. 2d. The local data result in a clear hysteresis where two sharp drops/jumps of the PEEM intensity are visible. Analogue to the changes in the overall $\mathrm{CO}_{2}$ reaction rate in dependence of the CO partial pressure as measured by MS, the drops/jumps in the PEEM intensity represent kinetic transitions from one reactivity state of the system to another, but measured locally for one particular grain of interest. The reason for the much more pronounced local transitions observed by PEEM (in comparison to MS) lies in the averaging effect of the MS measurements: the kinetic transitions of the differently oriented grains occur at different $\mathrm{CO}$ pressures, the corresponding drops/jumps in the $\mathrm{R}_{\mathrm{CO} 2}$ curve overlap, thus smearing out the global $\tau_{\mathrm{A}}$ and $\tau_{\mathrm{B}}$.

Again, similarly as in the global case, the treatment of the polycrystalline Pd surface with oxygen at elevated temperature (e.g. at $973 \mathrm{~K}$ as is shown in Fig. 3b) results in the collapse of the hysteresis loop, the local $\tau_{\mathrm{A}}$ and $\tau_{\mathrm{B}}$ transitions are smeared out and it is not possible to associate the transitions with particular $\mathrm{CO}$ pressure values as in the case of the clean Pd surface (Fig. 3a). The reason for this behavior will be discussed below, together with the XPS data.

\subsection{XPS Results}

To explain the observed reaction behavior, an XPS-analysis of the Pd foil has been performed before and after the oxidizing oxygen treatment at constant oxygen pressure of $5 \times 10^{-6}$ mbar and temperatures between 673 and $973 \mathrm{~K}$. The XPS results are summarized in Figs. $4 \mathrm{a}-\mathrm{c}$, in which the high-resolution spectra for the Pd 3d region (Fig. 4a), for the $\mathrm{Si} 2$ p region (Fig. 4b) and the evolution of the $\mathrm{Si} 2 \mathrm{p}$ signal with surface oxidation temperature (Fig. 4c) are shown. The Pd 3d spectra demonstrate that no significant formation of palladium oxide has been observed under the present conditions. In turn, the $\mathrm{Si} 2 \mathrm{p}$ spectra reveal a remarkable $\mathrm{SiO}_{2}$ formation on the $\mathrm{Pd}$ surface upon the oxidizing treatment at elevated temperatures. Apparently, the presence of oxygen stimulates the diffusion of bulk dissolved $\mathrm{Si}$ from the near-surface regions towards the surface and thus enhances the segregation of $\mathrm{Si}$ to the $\mathrm{Pd}$ surface (compare the $\mathrm{Si}^{0}$ and the $\mathrm{Si}^{4+} 2 \mathrm{p}$ signals, measured for the $\mathrm{Si}$ segregated without and with oxygen atmosphere). As 


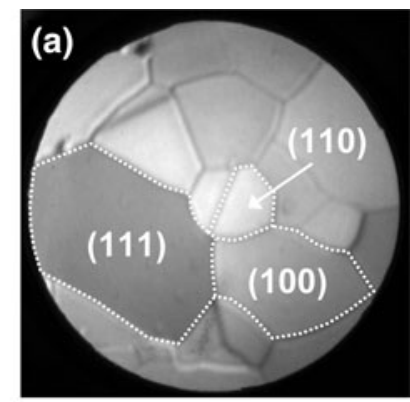

(d)

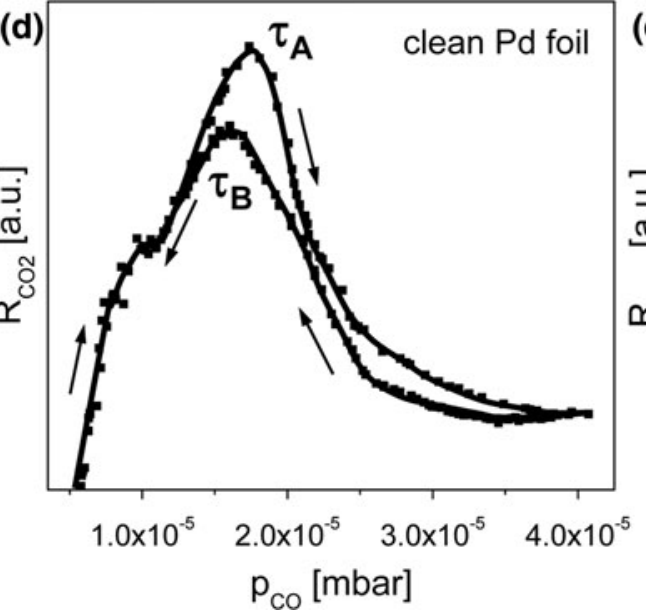

Fig. 2 CO oxidation on polycrystalline Pd foil. (a) PEEM image of the clean $\left(\mathrm{Ar}^{+}\right.$sputtered and annealed) $\mathrm{Pd}$ surface, three low index domains are exemplary indicated, (b) the same surface, but oxygen covered, (c) the same surface after oxidizing treatment at $\mathrm{po}_{2}=5 \times 10^{-6}$ mbar and $\mathrm{T}=973 \mathrm{~K}$, (d) global $\mathrm{CO}_{2}$ reaction rate for a clean Pd surface recorded by $\mathrm{MS}$ during an isothermal $(\mathrm{T}=473 \mathrm{~K})$ cyclic $\mathrm{CO}$ pressure scan at constant oxygen pressure

for any diffusion related process, the segregation limited $\mathrm{SiO}_{2}$ formation rate appears to be strongly temperature dependent, as demonstrated in Fig. 4c. An attempt to apply an Arrhenius plot provides an effective activation energy of $0.27 \mathrm{eV}$, a value which is significantly lower than known values of about $2 \mathrm{eV}$ for thermal Si oxidation [24, 25]. It is clear, however, that in the present case of a submonolayer $\mathrm{SiO}_{2}$, the Deal-Grove model [24] based on the assumption that Si oxidation proceeds by the transport of molecular oxygen from the ambient to the $\mathrm{Si} / \mathrm{SiO}_{2}$ interface through already oxidized $\mathrm{Si}$ layers does not apply. In our case, the rate limitation does not result from the oxygen diffusion, but from the silicon transport from the $\mathrm{Pd}$ subsurface region to the surface. Thus, the activation energy values typical for the oxygen diffusion limited process are not expected. Recently, unusually small activation energy values (of e.g. $0.13 \mathrm{eV}$ [25] or even lower [26]) were observed for the oxidation procedure where the oxygen diffusion limit was lifted by using atomic oxygen for $\mathrm{Si}$ oxidation.

Generally, experimental observations are known, which show that the presence of oxygen (even in the $10^{-6} \mathrm{mbar}$ range) increases the mobility of $\mathrm{Si}$ in Pt-metals causing the precipitation of $\mathrm{SiO}_{\mathrm{x}}$ at the surface [27]. The enhanced $\mathrm{Si}$
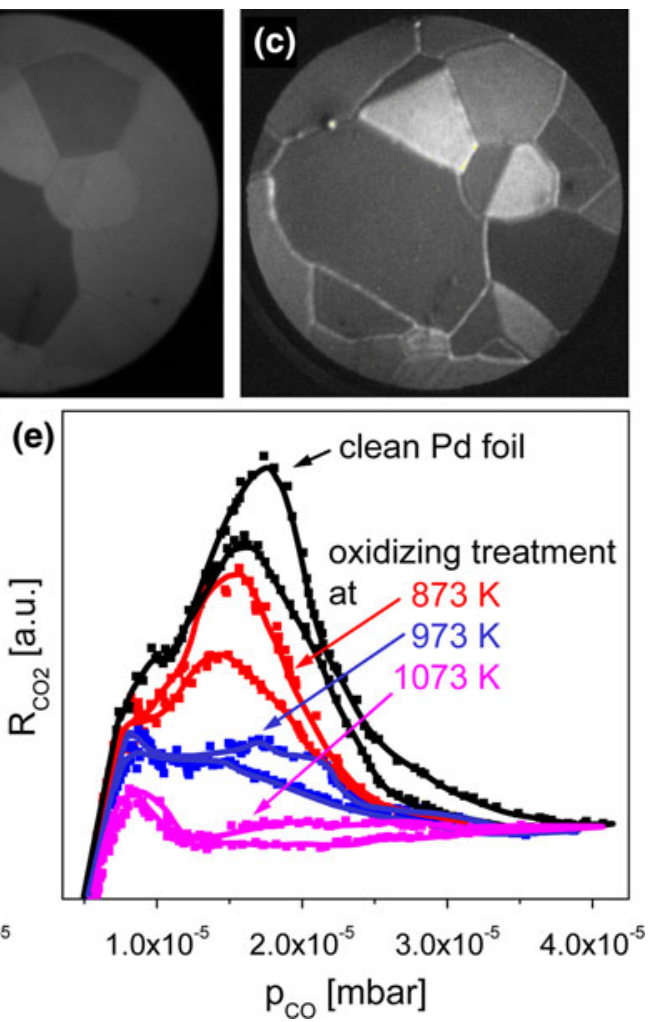

of $1.3 \times 10^{-5}$ mbar. Kinetic transitions $\tau_{\mathrm{A}}$ and $\tau_{\mathrm{B}}$ from the catalytically active to the inactive steady state and vice versa are indicated, (e) the same as in (d) but for the oxygen treated surface $\left(\mathrm{po}_{2}=5 \times 10^{-6}\right.$ mbar, $\mathrm{T}=873,973,1073 \mathrm{~K}$, treatment duration $15 \mathrm{~min}$ for all temperatures). $\mathrm{A} \mathrm{CO}_{2}$ reaction rate curve for the clean surface is also shown for comparison

segregation in the presence of oxygen can be rationalized considering energetic arguments: the higher bonding strength of Si on an oxygen covered Pd surface provides an energetic sink for $\mathrm{Si}$ atoms due to the chemical potential gradient, similarly as it occurs e.g. in an enrichment of alkali atoms on the oxygen covered surface in $\mathrm{CO}$ oxidation on $\mathrm{Rh}$ [28] or hydrogen oxidation on Rh [29, 30], or in the formation of concentration patterns of electropositive adsorbates as a result of up-hill diffusion in two-dimensional first order phase transitions under reaction-free conditions [31, 32]. Recently, DFT calculations were performed to identify the stable $\mathrm{Si}-\mathrm{O}$ bonding structures on the $\mathrm{Pd}(111)$ and $\mathrm{Pt}(111)$ surfaces [33], where a variety of stable intermediates with stoichiometries between $\mathrm{SiO}_{2}$ and $\mathrm{SiO}_{3}$, depending on the coverage, were found. This might explain the inhibiting effect of the $\mathrm{SiO}_{\mathrm{x}}$ growth on the $\mathrm{PdO}_{\mathrm{x}}$ formation observed in the present experiments, again due to the energetic arguments which favor the Si rather than the Pd oxidation.

The easiest interpretation of the inhibiting role of $\mathrm{SiO}_{2}$ in the $\mathrm{CO}$ oxidation reaction would be that $\mathrm{SiO}_{2}$ simply blocks a part of the active reaction sites on the Pd surface but does itself not participate in the reaction. However, the 


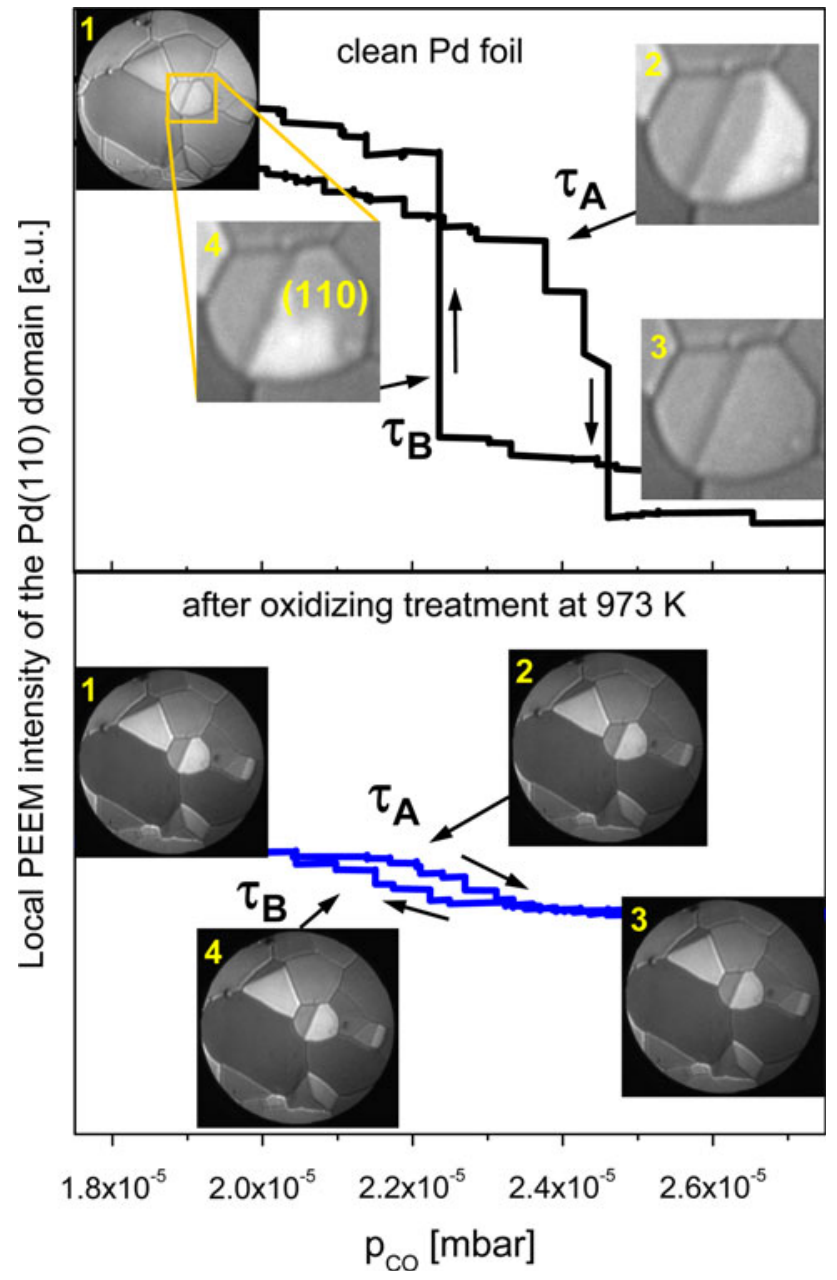

Fig. 3 Laterally-resolved kinetics of $\mathrm{CO}$ oxidation on individual grains of the Pd foil, (a) local intensity of the PEEM image for the $\mathrm{Pd}(110)$ domain recorded during the same cyclic CO pressure scan as in Fig. 2d. The kinetic transitions $\tau_{\mathrm{A}}$ and $\tau_{\mathrm{B}}$ are much more pronounced than in the global MS curve (Fig. 2d). The PEEM frames correspond to the reaction stages indicated in the PEEM intensity curve, (b) the same as in (a) but after the oxidizing oxygen treatment at $973 \mathrm{~K}$. The $\tau_{\mathrm{A}}$ and $\tau_{\mathrm{B}}$ points are almost indiscernible

drastic decrease in the $\mathrm{CO}_{2}$ formation rate for a rather small coverage of $\mathrm{SiO}_{2}$ (just a fraction of a monolayer, as follows from the $\mathrm{Pd} 3 \mathrm{~d}$ and $\mathrm{Si} 2 \mathrm{p}$ signal relation) requires an additional explanation. It is known that oxides on the Ptgroup metals form monolayer islands at the initial growth stage [22, 34]. The boundary lines between the oxide islands and the remaining "oxide-free" metal surfaces exhibit, due to the local electron density jump from the metal to the oxide surface, different electronic properties which may nanometer-wide extend the promoting effect along the free metal surface around the islands [22].

The promoting effect on the reaction observed in the case of $\mathrm{CeO}_{\mathrm{x}}$ islands, which is due to the additional oxygen supply [22], cannot be expected for $\mathrm{SiO}_{2}$ because of the poor redox properties of $\mathrm{SiO}_{2}$ and thus the absence of an
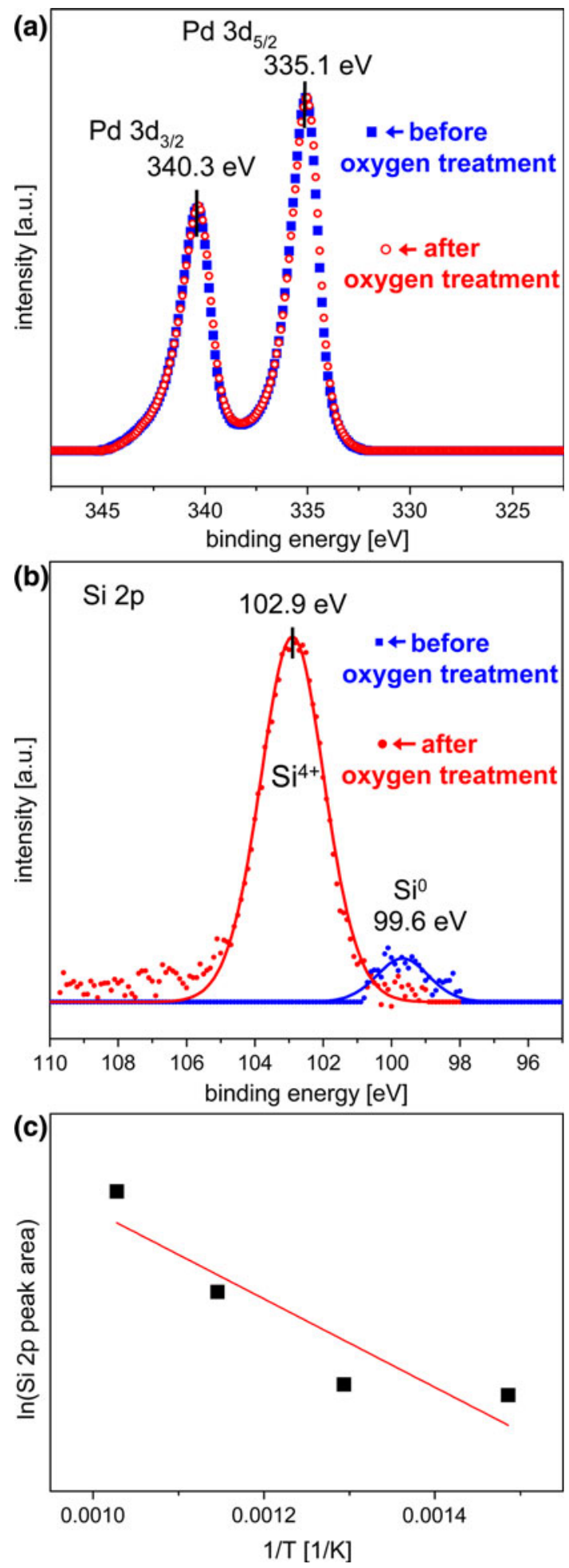

Fig. 4 Silicon oxide formation on Pd surface. (a) Pd 3d XPS spectra before and after oxidizing oxygen treatment at $973 \mathrm{~K}$, (b) the same but for the Si $2 p$ spectra, (c) Arrhenius plot

oxygen storage capacity. Whereas the refilling of the oxygen vacancies in reducible oxides such as $\mathrm{CeO}_{\mathrm{x}}$ stimulates the oxygen dissociation on the oxide-island adjacent 
surface sites, this is not the case for $\mathrm{SiO}_{2}$. In turn, the spillover of $\mathrm{CO}$ from the $\mathrm{SiO}_{2}$ islands (a weakly bound mobile $\mathrm{CO}$ layer can be formed on the $\mathrm{SiO}_{2}$ surface [35]) supports the interpretation that the regions around the $\mathrm{SiO}_{2}$ islands must be rather inactive for the $\mathrm{CO}$ oxidation.

\section{Summary}

A combined PEEM-MS-XPS approach allows us to study the in situ kinetics of surface reactions under control of the surface chemical composition of the sample, also under reactive atmospheric conditions in the $10^{-5}$ mbar range. This also allows to trace reaction induced compositional changes of the sample surface, e.g. as the result of segregation effects.

In the present study silicon oxide formation, resulting from Si-impurity segregation on the polycrystalline Pd-foil at oxidizing conditions in the temperature range between $873 \mathrm{~K}$ and $1073 \mathrm{~K}$, was monitored by XPS and its impact on the global and local (spatially-resolved) kinetics of the $\mathrm{CO}$ oxidation was determined by MS and PEEM. The results reveal a drastic inhibiting effect of silicon oxide on the $\mathrm{Pd}$ reactivity towards $\mathrm{CO}$ oxidation, manifested both in the collapse of the global $\mathrm{CO}_{2}$ formation rate (as measured by MS) and in the modified local reactive properties (as observed by PEEM) of individual Pd micrograins. The presence of adsorbed oxygen on the Pd surface effectively enhances the silicon segregation to the Pd surface, due to energetic reasons.

Acknowledgments Technical support by Johannes Frank (IMC, TU Vienna) is cordially acknowledged. This work was partially supported by the Austrian Science Fund (FWF) [SFB-F45-04 FOXSI] and by the ÖAD, Project 84ÖU5.

Open Access This article is distributed under the terms of the Creative Commons Attribution License which permits any use, distribution, and reproduction in any medium, provided the original author(s) and the source are credited.

\section{References}

1. Engel T, Ertl G (1978) J Chem Phys 69:1267

2. Ertl G (2008) Angew Chem Int Ed 47:3524 and references therein

3. Suchorski Y, Drachsel W (2007) Top Catal 46:201 and references therein
4. Sander M, Imbihl R, Ertl G (1992) J Chem Phys 97:5193

5. Lauterbach J, Haas G, Rotermund HH, Ertl G (1993) Surf Sci 294:116

6. Lauterbach J, Rotermund HH (1994) Catal Lett 27:27

7. Suchorski Y, Spiel C, Vogel D, Drachsel W, Schlögl R, Rupprechter G (2010) Chem PhysChem 11:3231, and supporting information therein

8. Vogel D, Spiel C, Suchorski Y, Urich A, Schlögl R, Rupprechter G (2011) Surf Sci 605:1999

9. Spiel C, Vogel D, Suchorski Y, Drachsel W, Schlögl R, Rupprechter G (2011) Catal Lett 141:625

10. Vogel D, Spiel C, Suchorski Y, Trinchero A, Grönbeck H, Schlögl R, Rupprechter G (2012) Angew Chem Int Ed 51:10041

11. Goodman DW, Peden CHF, Chen MS (2007) Surf Sci 601:L124

12. Gao F, Wang Y, Cai Y, Goodman DW (2009) J Phys Chem C 113:174

13. Gao F, Cai Y, Gath KK, Wang Y, Chen MS, Guo QL, Goodman DW (2009) J Phys Chem C 113:182

14. Gao F, McClure SM, Cai Y, Gath KK, Wang Y, Chen MS, Guo QL, Goodman DW (2009) Surf Sci 603:65

15. Rupprechter G, Weilach C (2008) J Phys Condens Matter 20:184019

16. Hendriksen BLM, Frenken JWM (2002) Phys Rev Lett 89:046101

17. Hendriksen BLM, Bobaru SC, Frenken JWM (2004) Surf Sci 552:229

18. van Rijn R, Balmes O, Felici R, Gustafson J, Wermeille D, Westerström R, Lundgren E, Frenken JWM (2010) J Phys Chem C 114:6875

19. Gao F, Wang Y, Goodman DW (2010) J Phys Chem C 114:6874

20. Zorn K, Giorgio S, Halwax E, Henry CR, Grönbeck H, Rupprechter G (2011) J Phys Chem C 115:1103

21. Suchorski Y, Wrobel R, Becker S, Strzelczyk B, Drachsel W, Weiss H (2007) Surf Sci 601:4843

22. Suchorski Y, Wrobel R, Becker S, Weiss H (2008) J Phys Chem C 112:20012

23. Bader S, Richter L, Orent T (1982) Surf Sci 115:501

24. Deal BE, Grove AS (1965) J Appl Phys 36:3770

25. Sekine K, Saito Y, Hirayama M, Ohmi T (2001) IEEE Trans Electr Dev 48:1550

26. Saito K, Jin Y, Ono T, Shimada M (2004) Jpn J Appl Phys 43:L765

27. Salmeron M, Somorjai GA (1981) J Vac Sci Techn 19:722

28. Suchorski Y, Block JH (1996) Appl. Surf Sci 94(95):200

29. Marbach H, Günther S, Luerssen B, Gregoratti L, Kiskinova M, Imbihl R (2002) Catal Lett 83:161

30. Marbach H, Lilienkamp G, Wie H, Günther S, Suchorski Y, Imbihl R (2003) Phys Chem Chem Phys 5:2730

31. Naumovets AG (1994) In: King DA, Woodruff DP (eds) The chemical physics of solid surfaces, vol 7. Elsevier, Amsterdam, p 163

32. Lyuksyutov I, Naumovets AG, Pokrovsky V (1992) Twodimensional crystals. Academic Press, Boston

33. Kershner DC, Hyman MP, Medlin JW (2008) Surf Sci 602:3603

34. Klein C, Kresse G, Surnev S, Netzer FP, Schmid M, Varga P (2003) Phys Rev B 68:235416

35. Blomqvist A, Lehman L, Salo P (2012) Phys Stat Sol 249:1046 